\title{
Zwischen Mitgestalten und Alltagsdruck: Herausforderungen zivilgesellschaftlich Engagierter zur (Wieder)belebung einer Kleinstadt in Ostdeutschland
}

\section{Between co-creation and daily routine: challenges of volunteering people for (re) vitalizing their small town in Eastern Germany}

https://doi.org/10.2478/rara-2020-0016

Eingegangen: 29. Juni 2019; Angenommen: 17. April 2020

Kurzfassung: Zivilgesellschaftliches Engagement steht gerade in Städten, die von Bevölkerungsrückgang betroffen sind, vor großen Herausforderungen, da soziale Netzwerke ausgedünnt und kommunale Dienstleistungen im freiwilligen Pflichtaufgabenbereich reduziert worden sind. Gleichzeitig nimmt in solchen Städten das zivilgesellschaftliche Engagement einen besonderen Stellenwert ein, um Engpässe bei der Daseinsvorsorge auszugleichen. Der Artikel untersucht die lebensweltlichen Umstände von zivilgesellschaftlich Engagierten und will damit einen Beitrag zum Verständnis für den unterschiedlichen Umfang des Engagements bestimmter Personengruppen in bestimmten Lebensphasen leisten. Anhand von Projektverläufen im zivilgesellschaftlichen Engagement, initiiert von jüngeren Personengruppen, werden Hemmnisse und Bedarfe aufgezeigt, die die Engagierten erfahren haben. Die Befunde zeigen, dass bürokratische und hierarchische Hürden, geringe Wertschätzung freiwilliger Arbeit und Interessenkonflikte zwischen Fördergebern und Engagierten von Letzteren als wesentliche Hemmnisse wahrgenommen werden. Dem gegenüber stehen Einzelfaktoren zivilgesellschaftlichen Engagements, die im Einklang mit den lebensweltlichen Umständen der Engagierten stehen, wie gemeinsames Arbeiten, ein offener, flexibler Rahmen für das individuelle Handeln wie auch dessen flexible Einteilung und Organisation. Dem Beitrag liegen empirische Daten zugrunde, die durch Einzelfallanalysen in der ostdeutschen Kleinstadt Weißwasser erhoben worden sind.

Schlüsselwörter: Zivilgesellschaftliches, Engagement, Bottom-up-Prozess, demographischer Wandel, strukturschwache Region, Weißwasser

Abstract: Civic engagement faces major challenges, especially in cities affected by declining populations, as social networks have been thinned out and services in the sector of voluntary municipal compulsory tasks have been reduced. At the same time, civic engagement is particularly important in such cities in order to compensate for bottlenecks in services of general interest. This paper examines the life worlds of voluntarily engaged people and aims

*Corresponding author: Dr. Olivia Kummel, ILS - Institut für Landes- und Stadtentwicklungsforschung, Brüderweg 22-24, 44135 Dortmund, Deutschland, E-mail: olivia.kummel@ils-forschung.de 
to contribute to an understanding of the different levels of involvement of certain groups of people in certain phases of life. On the basis of project histories in civic engagement, initiated by younger groups of people, the obstacles and needs experienced by those involved are highlighted. The findings show that bureaucratic and hierarchical hurdles, low appreciation of voluntary work and conflicts of interest between funding bodies and those involved are perceived by the latter as major obstacles. On the other hand, there are individual factors of civic engagement that are in harmony with the conditions of the life worlds of those voluntarily active, such as working together, an open, flexible framework for individual action and its flexible division and organisation. The paper is based on empirical data collected in individual case analyses in the small town Weißwasser in Eastern Germany.

Keywords: Civic engagement, Bottom-up process, Demographic change, Structurally weak region, Weißwasser

\section{Einleitung}

Zivilgesellschaftliches Engagement ist der Kitt, der eine Gesellschaft zusammenhält und das Gemeinwohl stärkt (Zimmer 2010: 180; BMFSFJ 2017: 3; Alscher/Priller/ Burkhardt 2018: 373). Zudem gilt zivilgesellschaftliches Engagement in seiner dem Gemeinwohl dienenden Absicht als einer der Grundpfeiler einer demokratisch orientierten Gesellschaft (Zimmer 2010: 180; Antonov 2016: 5; Simonson/Ziegelmann/Vogel et al. 2016: 15). Eine besondere Bedeutung kommt dem zivilgesellschaftlichen Engagement bei der Schaffung gleichwertiger Lebensverhältnisse zu, in der wachsenden Stadt wie in ländlichen Räumen (BBSR 2017: 7). Diese wurden zu Beginn der 2000er-Jahre stark in Frage gestellt und einer veralteten Förderpolitik zugeschrieben. Daraufhin wurde die finanzielle Unterstützung für periphere, strukturschwache Regionen zurückgefahren und zeigen sich in der Verschlechterung der Standards (Barlösius/Neu 2008: 21; Aring 2013: 44). Periphere ländliche Gemeinden sollten mehr selbstverantwortliche Handlungsspielräume bekommen, um so den Rückzug der staatlichen Gewährleistungsfunktionen und die Finanzknappheit der öffentlichen Hand zu kompensieren (vgl. Reim/ Schmithals 2008: 75). Vor allem in strukturschwachen, vom demographischen Wandel betroffenen Gemeinden werden auch zukünftig freiwillig Engagierte in die Erbringung von Daseinsvorsorgeleistungen eingebunden, die vorher teilweise staatlich organisiert waren (BBSR 2017: 7). Kleinstädte übernehmen hier wichtige Ankerfunktionen, um die Versorgung der dünn besiedelten Räume aufrechtzuerhalten.

Dass diese Überlegung zu kurz gegriffen ist, erscheint offensichtlich. So fehlen in vom demographischen Wandel stark betroffenen Regionen vor allem jüngere Bevölkerungsgruppen, in denen ein wesentliches Potenzial an zivilgesellschaftlichem Engagement vermutet wird. Die aufgrund des Bevölkerungsrückganges stark ausgedünnten sozialen Netzwerke können oft die eigenverantwortliche Organisation von zivilgesellschaftlichem Engagement kaum mehr übernehmen (vgl. Olk/Gensicke 2014: 207; Nadler 2017: 505). So ist es nötig, ein breiteres Verständnis für die Unterschiede im Engagement bestimmter Personengruppen bzw. deren Lebensphasen zu entwickeln (Nadler 2017: 507; Kummel/Nadler 2018: 59). Genau hier setzt dieser Beitrag an: Es werden anhand von Projektverläufen im zivilgesellschaftlichen Engagement, initiiert von jüngeren Personengruppen, Hemmnisse und Bedarfe aufgezeigt, die die Engagierten während ihrer Projektarbeit erfahren haben. Im Beitrag wird der Frage nachgegangen, wie zivilgesellschaftliches Engagement in einer vom demographischen Wandel stark betroffenen Stadt ermöglicht und Eigeninteressen stärker eingebracht werden können.

Dazu werden im folgenden Kapitel zunächst die Rahmenbedingungen für zivilgesellschaftliches Engagement in Deutschland dargestellt und exemplarisch der Kontext für stark vom demographischen Wandel betroffene ländliche Regionen Ostdeutschlands und dessen mögliche Engagementpotenziale erläutert. Das dritte Kapitel befasst sich mit den empirischen Befunden einer Einzelfallstudie, die im Rahmen einer dreijährigen Begleitung von Engagierten in der stark geschrumpften Kleinstadt Weißwasser in der Oberlausitz (Sachsen) erhoben worden sind. Diese werden im vierten Kapitel diskutiert. Im Fazit werden Möglichkeiten aufgezeigt, zivilgesellschaftliches Engagement mit der Lebenswelt der Engagierten in Einklang zu bringen und zu fördern. 


\section{Zivilgesellschaftliches Engagement und demographischer Wandel}

Da es für zivilgesellschaftliches Engagement unterschiedliche Begriffsverständnisse gibt, beziehe ich mich in diesem Beitrag im Wesentlichen auf fünf Dimensionen, die mit diesem Begriff abgedeckt werden.

- Unter zivilgesellschaftlichem Engagement versteht man eine freiwillige, unbezahlte, gemeinwohlorientierte Arbeit (Deutscher Bundestag 2002: 24; BAGFA 2014: 2; Antonov 2016: 9; Priller 2016: 162; Alscher/ Priller/Burkhardt 2018: 373).

- Zivilgesellschaftliches Engagement findet im öffentlichen Raum statt, also in zivilgesellschaftlichen Organisationen oder im weniger organisationsgebundenen Rahmen (Antonov 2016: 8; Priller 2016: 162; Alscher/Priller/Burkhardt 2018: 373).

- Als zivilgesellschaftliches Engagement wird eine Tätigkeit verstanden, die nicht auf berufliche Qualifikationen abzielt (Antonov 2016: 8; Nadler 2017: 501).

- Mit zivilgesellschaftlichem Engagement wird keine direkte politische Macht ausgeübt (Nadler 2017: 500).

- Zivilgesellschaftliches Engagement, in das eigene Ideen eingebracht werden können, wird autonom und selbst geführt (BAGFA 2014: 2; Antonov 2016: 8).

Anhand dieser fünf Dimensionen soll im Folgenden näher erläutert werden, ob in den vom demographischen Wandel betroffenen Städten zivilgesellschaftliches Engagement möglicherweise anders verhandelt wird, da die Rahmenbedingungen aufgrund von Mangel an verschiedensten Ressourcen anders gelagert sind. Zunächst wird der Stand des zivilgesellschaftlichen Engagements in Deutschland im Allgemeinen erläutert, um anschließend die Rahmenbedingungen in den vom demographischen Wandel betroffenen ländlichen Räumen in Ostdeutschland zu betrachten.

\subsection{Zivilgesellschaftliches Engagement in Deutschland}

Die Bundesregierung sieht in der Engagementpolitik eine Schwerpunktaufgabe und hat 2016 die Engagementstrategie auf den Weg gebracht, um zivilgesellschaftliches Engagement stärker zu fördern und günstigere Rahmenbedingungen zur Entfaltung des Engagements zu schaffen (BMFSFJ 2017: 6). Damit unterstützt die Bun- desregierung die gestiegene Zahl an freiwillig Engagierten. Laut letztem Freiwilligensurvey in 2014 ist der Anteil Engagierter im Vergleich zur Erhebung in 2009 um 7,7 \% auf $43,6 \%$ deutschlandweit gestiegen. Seit Beginn der Erhebungen im Jahr 1999 ist diese Zahl besonders in den letzten fünf Jahren stark angestiegen. Dies kann zum einen am gestiegenen Bildungsniveau, zum anderen an der vermehrten Bewusstseinsbildung durch Politik und Öffentlichkeit liegen (Simonson/Ziegelmann/Vogel et al. 2016: 15-16).

Ein weiterer Grund für den gestiegenen Anteil an Engagierten ist ferner die Diversifizierung von Engagementformaten (Krimmer 2015: 5), wie Freiwilligenagenturen, Mehrgenerationenhäuser, Bürgerstiftungen oder auch zeitlich begrenzte, themenbezogene Initiativen (Gundert 2010: 110), die ein zusätzliches Angebot zum traditionellen Ehrenamt anbieten. Viele staatliche Programme unterstützen zivilgesellschaftliche Initiativen, die sich für das Gemeinwohl einsetzen. Häufig wird dabei Engagement mit der Auflage unterstützt, Partnerschaften mit Bürgerinnen und Bürgern, Kommunen und Staat einzugehen (Gundert 2010: 110; Klie/Marzluff 2012: 749; o.V. 2018: 27). Bei der Zusammenarbeit zwischen Bürgerinnen/Bürgern und Kommunen gibt Nadler (2017: 507) jedoch zu bedenken, dass sich Verwaltung und Politik häufig als die einzig legitimen Institutionen sehen, gemeinwohlorientierte Interessen zu definieren und durchzusetzen. Ein Mitgestalten der Stadtgesellschaft ist dann schwierig, wenn Daseinsvorsorgeleistungen von Engagierten erbracht werden sollen. Die Gefahr besteht darin, dass die Aufgaben, die von Bürgerinnen und Bürgern übernommen werden sollen, nicht ihren Eigeninteressen entsprechen und sie sich dann weniger einbringen wollen (Nadler 2017: 507). Generell wird von vielen Engagierten mehr Eigenverantwortung und ein Abbau bürokratischer Hürden gefordert (Kummel/Nadler 2018: 59). Auch der Freiwilligensurvey 2014 belegt mit Zahlen, dass sich über die Hälfte der Engagierten für mehr Information und Beratung zum Engagement wie auch für eine Unterstützung bei der Ausstattung mit einfachen Ressourcen wie Räumlichkeiten und Ausstattungsmitteln aussprechen (Simonson/Ziegelmann/Vogel et al. 2016: 21). Krimmer (2015: 5) fordert deshalb einen ermöglichenden Staat, der die Selbstorganisation und den Eigensinn im Engagement fördert.

Zivilgesellschaftliches Engagement ist nicht in allen Regionen Deutschlands gleich verteilt. Gerade in den vom demographischen Wandel betroffenen Regionen nimmt der Anteil engagierter Menschen ab, da durch die hohen Bevölkerungsverluste oft die kritische Masse an Engagementpotenzial zur Selbstorganisation fehlt 
(Nadler 2017: 505). Gleichzeitig wird in diesen Regionen ein erhöhter Bedarf an zivilgesellschaftlichem Engagement festgestellt, dem vor allem durch Anreize und Unterstützung selbstorganisierter Hilfeleistungen begegnet werden kann (ARL 2016: 33).

\subsection{Demographischer Wandel in Ostdeutschland}

Der demographische Wandel in Ostdeutschland ist, vor allem in ländlichen Räumen und deren Kleinstädten, durch massive Bevölkerungsverluste gekennzeichnet. Bei der jüngeren Bevölkerung zwischen 19 und 30 Jahren sind die Wanderungsverluste am höchsten.

Festzustellen ist ein West-Ost-Gefälle im Anteil der Engagierten. Während sich im Jahr 2014 in Westdeutschland 44,8 \% der Bevölkerung engagierten, waren es in Ostdeutschland 38,5\% (Kausmann/Simonson 2016: 564). Diese Unterschiede begründen Nadler (2017: 501) wie auch Olk und Gensicke (2014: 19) zum einen damit, dass während der DDR-Zeit die Zivilgesellschaft oft für politische Zwecke instrumentalisiert wurde, zum anderen durch eine hohe Arbeitslosigkeit, gefolgt von massiven Abwanderungsprozessen nach der Wiedervereinigung, die vor allem ländliche Regionen stark ausdünnten und Familienstrukturen aufbrachen. Olk und Gensicke (2014: 203) weisen in diesem Zusammenhang darauf hin, dass Engagement in strukturschwachen Regionen Ostdeutschlands eine andere Sinnzuschreibung erfährt. Gerade von den Verbliebenen wird Engagement als soziale Integration oder als Verbesserung der Qualifikation und Beschäftigung bei Arbeitslosigkeit betrachtet wie auch als Wertschätzung, Anerkennung und Familienersatz von älteren Menschen genutzt (Olk/ Gensicke 2014: 178). Infolgedessen unterscheiden sich auch die Engagementstrukturen dahingehend, dass in Westdeutschland eher formale Vereinsstrukturen bevorzugt werden, während in Ostdeutschland das informelle oder private Engagement, wie gegenseitige Hilfeleistungen im Familien-, Freundes- und Nachbarschaftskreis, verbreitet ist (Reim/Schmithals 2008: 78; Olk/Gensicke 2014: 12; Kausmann/Simonson 2016: 569-570). Letzteres wird von den informell Engagierten oft selbst nicht als solches wahrgenommen und taucht demnach in den Statistiken kaum auf (Kummel/Nadler 2018: 56).

Eine weitere Erklärung für unterschiedliche Engagementquoten wird in der ungleichen Verteilung von Engagement ermöglichenden Einrichtungen gesehen. Bischoff und Rahn (2015: 12) sprechen hier von einem West- Ost- wie auch von einem Süd-Nord-Gefälle.
Sittler, Amrhein und Krimmer (2015: 49) schlagen deshalb Engagement-Förderregionen vor, die sowohl kommunale, gesellschaftliche, demographische und wirtschaftliche Rahmenbedingungen einbeziehen wie auch informelles Engagement mit einbetten, um lokale organisationsübergreifende Engagementstrategien zu entwickeln, die dann autonom und ohne staatlich steuernde Einflüsse umgesetzt werden.

Zivilgesellschaftliches Engagement kommt somit der Aufgabe nach, auch in strukturschwachen Regionen zur Lebensqualität beizutragen. Die Bewertung von Lebensqualität hängt vor allem vom gesellschaftlichen Zusammenhalt ab (Küpper/Steinführer 2017: 52). Klie und Marzluff (2012: 748) wie auch Kummel und Nadler (2018: 59) merken dazu jedoch kritisch an, dass zivilgesellschaftliches Engagement kein Allheilmittel ist, dem demographischen Wandel und seinen Folgen zu begegnen. Vielmehr sei es nötig, Grenzen zu erkennen und die Rolle der Mitgestalterinnen und Mitgestalter neu zu definieren. Steinführer, Küpper und Tautz (2012: 5 ) berichten, dass vor allem in der kulturellen, gemeinschaftsstiftenden Daseinsvorsorge zivilgesellschaftliches Engagement eine wichtige Rolle einnimmt. Allerdings sind Engagierte gerade in stark geschrumpften Kleinstädten auf unterstützende Strukturen, wie Verlässlichkeit in der Planung und Ressourcen, angewiesen, um eine Kontinuität im Engagement zu fördern (Steinführer/Küpper/Tautz 2012: 5). Im Raumordnungsbericht 2017 (BBSR 2017: 54) wie auch von Gundert (2010: 115) wird ebenfalls empfohlen, eine „Unterstützungskultur“ zu pflegen, administrative Hürden für Engagement niedrig zu gestalten und bestimmte Bevölkerungsgruppen in ihren Lebenszusammenhängen zu aktivieren.

Besondere Bedeutung kommt den Kleinstädten als Ankerfunktion in den ländlichen Regionen zu, die von Bevölkerungsverlusten und starker Überalterung betroffen sind. In ländlichen Kleinstädten kann die Grundversorgung an Daseinsvorsorgeleistungen meist noch erfüllt werden. Dennoch hemmen auch hier nicht mehr vorhandene öffentliche Infrastrukturen freiwilliges Engagement, weil Ansprechpartnerinnen/-partner und Ressourcen fehlen.

\subsection{Engagementpotenziale in Kleinstädten Ostdeutschlands}

Politische Umbrüche, wie sie in Ostdeutschland erlebt wurden, erfordern bestimmte Strategien, um sich neu zu orientieren oder zu überleben. Willisch (2011: 83) beschreibt in diesem Zusammenhang eine Reduktion 
auf das Wesentliche als Überlebens- oder Schrumpfungsarrangements: Vom Kosten Verursachenden wird sich verabschiedet, alles Nützliche wird zusammengehalten. Visuell kommen diese Schrumpfungsarrangements durch brachgefallene, ehemals bedeutsame, identitätsstiftende soziale Infrastrukturen wie Gemeinschaftshäuser und Schulen drastisch zum Ausdruck (Steinführer/Moser 2016: 265). Als "Leitfiguren“ und „Kümmerer" werden solche Personen betitelt, die durch ihr Engagement alternative Lösungen für diese brachgefallenen Gemeinschaftseinrichtungen aufzeigen und weitere Personen für ihre Ideen begeistern können. Die Neuaneignung in Eigenverantwortung hat auch symbolischen Charakter: Staatliche Funktionen werden nicht ersetzt, sondern neuen Gegebenheiten angepasst und positiv in Wert gesetzt (Steinführer/Moser 2016: 273).

Stellt sich die Frage, wer diese Entfaltungsräume in ostdeutschen Kleinstädten in ländlichen, strukturschwachen Räumen nutzt, um eigene Ideen von zivilgesellschaftlichem Engagement umzusetzen. Schaut man sich die empirischen Befunde des Freiwilligensurveys 2014 an, so ist zu erkennen, dass sich bestimmte Bevölkerungsgruppen unterschiedlich stark engagieren. Frauen sind geringfügig weniger engagiert $(41,5 \%)$ als Männer (45,7\%). Unter den Bevölkerungsgruppen im Alter zwischen 14 und 29 Jahren (46,9\%) und zwischen 30 und $49(47,0 \%)$ ist das Engagement weitaus stärker etabliert als bei den über 65-Jährigen (34,6 \%) (Vogel/ Hagen/Simonson et al. 2016: 93).

Bereits aus dem ersten Engagementbericht von 2012 geht hervor, dass vor allem Frauen in bestimmten Bereichen wie im Sport oder in Leitungspositionen unterrepräsentiert sind. Zudem ist ihr Engagement in den Lebensphasen unterschiedlich ausgeprägt. Während junge Frauen im Alter zwischen 25 und 30 Jahren im öffentlichen Engagement weniger aktiv sind als Männer, unterscheidet sich die Engagementquote im Alter zwischen 45 und 55 Jahren kaum zwischen den Geschlechtern (BMFSFJ 2012: 13). Einen weiteren Grund für das geringere Engagement bilden die geringen Verwirklichungsräume qualifizierter junger Frauen in ländlich-peripheren Regionen Ostdeutschlands. Mit ihren kreativen Potenzialen geben jedoch gerade sie Impulse für neue Formen zivilgesellschaftlichen Engagements (Gabler/Kollmorgen/Kottwitz 2016: 5). Das Engagement der Jugendlichen im Alter zwischen 14 und 29 Jahren hat in den letzten fünf Jahren stark zugenommen, vor allem bei Schülerinnen und Schülern, was damit begründet wird, dass vermehrt ein höherer Bildungsabschluss angestrebt wird. Zivilgesellschaftliche Organisationen haben das Engagementpotenzial der Jugendlichen erkannt und aktivieren sie gezielt (Vogel/Hagen/Simonson et al. 2016: 94). Nicht zuletzt ist freiwilliges Engagement für Schülerinnen und Schüler attraktiv, wenn sie dadurch Vorteile für ihre spätere Berufswahl erzielen (BMFSFJ 2012: 14). Auch die Enquete-Kommission „Zukunft der ländlichen Regionen vor dem Hintergrund des demografischen Wandels" sowie Mai und Swiaczny (2008: 15) teilen die Meinung, dass die Aktivierung Jugendlicher zur nachhaltigen Entwicklung ländlicher Räume unerlässlich ist, allerdings brauche es alternative Formate und feste Ansprechpartnerinnen/-partner, um ein kontinuierliches Engagement $\mathrm{zu}$ ermöglichen (Landtag Brandenburg 2018: 85-86).

Als weiteres Engagementpotenzial in Kleinstädten Ostdeutschlands lassen sich Personen mit einer Zuwanderungsgeschichte beschreiben. Diese weisen zwar aufgrund sozialstruktureller Faktoren ein niedrigeres langfristiges Engagement auf (BMFSFJ 2012: 13), verfügen jedoch über Erfahrungen von anderswo, die sie an ihrem neuen Wohnort einbringen können.

Vor allem gilt es, diese drei Bevölkerungsgruppen mit ihren bislang in zu geringem Umfang eingebrachten Engagementpotenzialen zukünftig in stärkerem Maße einzubinden. Von der Fachwelt werden dazu Bottomup-Partnerschaften vorgeschlagen, um bestimmte zivilgesellschaftliche Gruppen zu aktivieren, Sozialkapital zu entwickeln und regionale Kooperationen zu fördern (Beetz/Huning/Plieninger 2008: 296; Küpper/Kundolf/ Mettenberger et al. 2018: 231). Aus diesen Bottom-upProzessen können Strategien entwickelt werden, die in Kleinstädten einen kollektiven und kollaborativen Umgang mit den Folgen der Schrumpfung anregen.

\section{Zivilgesellschaftlich Engagierte in einer schrumpfenden Kleinstadt Ostdeutschlands}

\subsection{Das Fallbeispiel Weißwasser}

Im Rahmen der Begleitforschung für das Projekt „Kleinstadt macht Leute, Leute machen Kleinstadt: Innovative Anreizsysteme für aktive Mitgestaltung im demografischen Wandel am Beispiel Weißwasser/Oberlausitz" („Kleinstadt_gestalten“), gefördert durch das Programm "Kommunen innovativ" vom Bundesministerium für Bildung und Forschung (BMBF), wurden acht Bürgerprojekte von Engagierten ins Leben gerufen, die hauptsäch- 
lich Bedarfe in der kulturellen Daseinsvorsorge abdeckten. Das Projekt war in Weißwasser als Referenzstadt angesiedelt und hatte eine Laufzeit von 2016 bis 2019. Das Ziel des Projektes bestand darin, die spezifischen Bedürfnisse der Bevölkerungsgruppen Jugendliche, junge Frauen und Rückkehrer/Zugezogene zu erforschen, die Anreiz- und Anerkennungskultur für deren Engagement auszubauen und für sie mehr Möglichkeitsräume zu schaffen. Mit dem Projekt war als Ziel die Erprobung von Anreiz- und Aktivierungsmechanismen für die drei oben genannten Zielgruppen verbunden, um anschließend die Bürgerprojekte in die Verstetigung zu führen und ein kontinuierliches Engagement sicherzustellen. Im Rahmen des Projekts „Kleinstadt_gestalten" wurden die Bürgerprojekte durch zwei Mitarbeiter in einem Vor-Ort-Büro und durch einen Kleinprojektefonds unterstützt, der von der Stadtverwaltung Weißwasser verwaltet wurde.

Weißwasser ist eine ehemalige Industriestadt, groß geworden durch die Glas- und Montanindustrie. Auch heute noch bestimmen diese beiden Industriezweige einen Teil der kleinstädtischen Wirtschaft, jedoch längst nicht mehr in dem Umfang wie zu DDR-Zeiten. Die Kleinstadt im äußeren Nordosten Sachsens ist umgeben von einem dünn besiedelten, strukturschwachen Raum und liegt an der Grenze zu Brandenburg und Polen. Weißwasser wird vor allem durch eine touristisch genutzte Landschaft im Norden eingerahmt: dem grenzüberschreitenden UNESCO Global Geopark „Muskauer Faltenbogen" und dem Fürst-Pückler-Park Bad Muskau samt Schloss. Im Süden grenzt der noch aktive Braunkohlentagebau „Nochten“ an die Stadtgrenze. Nach der Wiedervereinigung hatte die Stadt massive Abwanderungen zu verzeichnen, vor allem von jüngeren Menschen. Über die Hälfte der Bevölkerung verließ seit 1990 die Stadt, sie schrumpfte von rund 38.000 Einwohnern 1989 auf rund 16.000 Einwohner im Jahr 2018 (Kummel/ Nadler 2018: 54).

Vor allem das Städtebauförderprogramm „Stadtumbau Ost" veränderte den Stadtgrundriss. Das Neubaugebiet "Weißwasser Süd“, in dem zu DDR-Zeiten $67 \%$ der Einwohnerinnen/Einwohner lebten, wurde fast komplett rückgebaut und wieder aufgeforstet (Stölzel 2019: 22). Vor allem den kommunalen Wohnungsunternehmen sollte durch die „Entlastung sogenannter DDRAltschulden ein starker Beteiligungsanreiz" geboten werden (Steinführer/Moser 2016: 271), was eher einer „Entrepreneuralisierung“ im Sinne der Anpassung statt Wachstum gleichkam (Bernt 2009: 755). Bernt, Kabisch und Peter (2005: 54) beschrieben die Wahrnehmung des massiven Schrumpfungsprozesses noch wie folgt:
„Der Niedergang Weißwassers hat bei der Bevölkerung zu einer Dominanz von Verlustmotiven in der Wahrnehmung der Stadtentwicklung geführt. Die Suche nach Zukunftsoptionen wird dadurch blockiert." Für die Bewohnerinnen und Bewohner, die in Weißwasser-Süd lebten und aufgewachsen sind, ist mit dem kompletten Abriss großer Teile des Stadtgebietes ein Stück Heimat verloren gegangen. Somit brachte der Stadtumbau zwar eine ökonomische Sanierung, gab aber den ehemaligen Bewohnerinnen und Bewohnern kaum gestalterischen Spielraum, den Stadtumbauprozess aktiv mitzugestalten (Bernt/Kabisch/Peter 2005: 51; Beetz/Huning/Plieninger 2008: 296; Bernt 2009: 763-765). Durch die finanziellen Engpässe der Stadtverwaltung wurden bei den freiwilligen Aufgaben im Kulturbereich Einsparungen vorgenommen. Zudem zog sich 2016 der Energieversorger Vattenfall $A G$ aus der Region zurück. Viele Kommunen in der Region hatten dadurch Millioneneinbußen zu verzeichnen. So musste Weißwasser für die vergangenen Jahre fast zehn Millionen Euro Gewerbesteuer zurückzahlen, da der Konzern Verluste zu verzeichnen hatte (Moeritz 2016: o.S.). Seit Kurzem schwächt sich jedoch die negative Bevölkerungsentwicklung ab (Weißwasser 2020). ${ }^{1}$ Ein Rückkehrer fasst seine Wahrnehmung zu Weißwassers Wandel in den 15 Jahren seiner Abwesenheit folgendermaßen zusammen:

\begin{abstract}
„Also von dem, was ich so mitbekommen habe, sind die Leute ein bisschen optimistischer geworden. Also vor sieben, acht Jahren ist man hier durch die Stadt gegangen, da wollte man wirklich nur noch weg. Weil alle grimmig geguckt haben. Das hast du denen angesehen, die hatten irgendwie keine Freude, [...] Ich meine, es ist klar, hier haben sie die halbe Stadt weggerissen. [...] Das waren massive Veränderungen, mit denen die hier klarkommen mussten. Und eigentlich konstant seit der Wende haben hier ja die Veränderungen angehalten. Und jetzt ist es, glaube ich, in einem Stadium, wo ein bisschen Ruhe reinkommt. Wo die großen, massiven Veränderungen durch sind erst einmal. Also die richtig großen. Und wo die Leute auch wieder ein bisschen, ja, einen Lichtblick haben. [...] Es entwickeln sich wieder Ideen und die haben vielleicht auch mehr Perspektiven oder, auf lange Sicht gesehen, einfach wieder einen optimistischeren Blick in die Zukunft. Also das hat sich wirklich doll geändert" (Rückkehrer 06, 05.02.2018). ${ }^{2}$
\end{abstract}

1 Vgl. https://www.weisswasser.de/zahlen_fakten (18.03.2020).

2 Die Interviews der Zugezogenen und Rückkehrer wurden transkribiert und anonymisiert. Die Transkripte wurden den jeweiligen drei Zielgruppen des Projektes zugeordnet, wie ,junge Frauen“, „Jugendliche“ und „Rückkehrer", und mit einer Nummer versehen. 


\subsection{Methodisches Vorgehen}

Die acht Bürgerprojekte und die Neuauflage eines zuvor bereits angedachten Bürgerprojektes wurden als Einzelfallanalyse in einer ethnographischen Studie (vgl. Flick 2012) mit teilnehmender Beobachtung (vgl. Patton 2002) wie auch mit Einzel- und Gruppeninterviews wissenschaftlich begleitet. Es wurde die ethnographische Methodik gewählt, da mit ihr Einblicke in die Alltagswelt der Engagierten gegeben werden können, um deren spezifischen Bedürfnisse herauszustellen und deren Möglichkeitsräume auszuloten, um herauszufinden, unter welchen Bedingungen ein langfristiges Engagement möglich erscheint. In einem Feldtagebuch wurden die Beobachtungen und Projektgespräche mit den Engagierten, Projektmitarbeiterinnen/-arbeitern und Bürgerinnen/Bürgern Weißwassers festgehalten. Zu Beginn und zum Abschluss wurden mit den Engagierten je sieben teilstandardisierte Leitfadeninterviews $(n=14)$ und während der Projektdurchführung drei zweistündige Gruppeninterviews mit den Initiatoren der Bürgerprojekte durchgeführt. Während der Projektlaufzeit gaben drei Engagierte ihre Bürgerprojekte auf oder gaben sie weiter an ihre Nachfolger. Die Gründe für ihr Ausscheiden wurden ebenfalls in Leitfadeninterviews erfasst. Die Leitfadeninterviews, die als Einzelinterviews geführt wurden, umfassten eine Dauer von 24 bis 68 Minuten. Sämtliche Interviews wurden mit dem Einverständnis der Engagierten aufgezeichnet und anschließend transkribiert. Ausgewertet wurden die Feldtagebuchnotizen und Transkripte der Einzel- und Gruppeninterviews mithilfe der qualitativen strukturierten Inhaltsanalyse (vgl. Mayring 1994), um sie verstehend zu interpretieren und so ein realistisches Bild der Engagierten zu zeichnen. Anschließend wurden die Ergebnisse zielgruppenspezifisch zusammengefasst und verglichen. Folgende Fragen waren dabei erkenntnisleitend: Welche hindernden und fördernden Aspekte beeinflussten den Projektverlauf? Welche Strategien verfolgten die Engagierten zur Umsetzung ihrer Projekte? Wie integrierten sie ihr Engagement in ihre Alltagswelt?

Aufgrund der räumlichen Selektivität lässt die Analyse keine Verallgemeinerungen zu, sondern zeigt Aktivierungs- und Handlungsmuster während des Umsetzungsprozesses der Bürgerprojekte auf. Diese sind spezifisch für den Fall Weißwasser und nur unter ähnlichen Ausgangsbedingungen auf andere Städte und städtische Gesellschaften übertragbar. Im Wesentlichen können durch Systematisierung des Datenmaterials vier Anreizoptionen identifiziert werden: Engagement als professionelle Weiterqualifizierung, Engagement als
Jobgenerator, Engagement und Eigeninteressen, Engagement und Gemeinwohl.

\section{3 „Die Zeit ist das Problem“ - Anreizoptionen für die Engagierten}

Der empirische Teil des Beitrages widmet sich der Darstellung der Alltagswelt der Engagierten und der Umsetzung ihrer Bürgerprojekte. Anhand der Kategorien Lebenshorizonte, Aktivierung zum Engagement, Bedarfsorientierung, Projektverlauf, Unterstützung und Projektverstetigung, die aus dem Datenmaterial selektiert wurden, werden die in Kapitel 3.2 genannten vier Anreizoptionen, sich zu engagieren, eingehender beleuchtet.

\subsubsection{Engagement als professionelle Weiterqualifizierung}

Viele Engagierte nutzen ihre berufliche Qualifikation und setzen ihr Wissen auch im freiwilligen Engagement ein. Es gibt aber auch Engagierte, die, noch neu in ihrem Job, das freiwillige Engagement als professionelle Weiterqualifizierung sehen, um ihre beruflichen Chancen zu erweitern. Vor allem zwei junge männliche Rückkehrer, die jeweils ein Studium erfolgreich abgeschlossen und noch keine eigene Familie gegründet hatten, hatten jeweils ihr eigenes Bürgerprojekt an den Start gebracht, allerdings mit unterschiedlichen Bedarfsorientierungen. Bens ${ }^{3}$ Bürgerprojekt war stark auf den kulturellen Bereich ausgerichtet, da er auch halbtags im Kulturbereich angestellt war und so Beruf und Engagement verbinden konnte:

Es ist sozusagen 'n Teil meiner Arbeit, die ich dort mache, den Kulturraum oder das Konzept für den Kulturraum zu überarbeiten. [...] Also, da kann man die Ansatzpunkte wunderschön verknüpfen. [...] Also ich versuche, das dann einfach pragmatisch zu sehen. Wenn ich dann einmal mit jemandem am Tisch sitze, den ich halt genau dann habe, warum soll ich den nicht noch auf was anderes ansprechen? [...] Und es bringt mir ja auch für die Arbeit dann wieder ' $n$ Mehrwert, weil dieses Netzwerk aufbauen steht ja auch bei uns mit drin in der Konzeption und deswegen ist das ja 'ne Sache, die so ' $n$ bisschen ineinandergreift und deswegen denke ich mal, hat man auch 'n ganz guten Ansatzpunkt (Ben, 30.01.2017).

3 Die Namen der Engagierten wurden durch fiktive Namen ausgetauscht, um deren Persönlichkeit zu schützen. 
Tims Bürgerprojekt widmete sich der Diversifizierung der sozialen Infrastruktur im Bereich der Kinderbetreuung. Er verband ebenfalls sein Engagement sehr effizient mit seinem Beruf als Projektmitarbeiter im soziokulturellen Bereich und als professionelle Weiterqualifizierung. Allerdings waren seine Anreizoptionen nicht primär beruflicher Natur, sondern auch stark getrieben vom Gemeinwohl und dem Ziel, eine Entlastung für Alleinerziehende zu schaffen und somit zur Verbesserung ihrer Lebensbedingungen beizutragen.

\begin{abstract}
„Also, es motiviert mich auch, weil's ja schon mit meiner Arbeit zu tun hat, ne? Also, es motiviert mich, weiterhin das zu tun, was ich jetzt eigentlich schon tue, also, dass ich für mich und meine Zukunft auch sage, das macht mir Spaß, ne, find's toll, wenn man Menschen hilft, denen 's eben nicht so gut geht, oder die halt, die's schwierig haben im Leben, und das würde mich einfach weiter motivieren, dass ich genau da weiter so an der Stange bleibe, und ist halt 'ne persönliche Motivation für mich und meine Arbeit und was ich tue, genau" (Tim, 18.01.2017).
\end{abstract}

Beide wurden in ihrem Engagement unterstützt von ihren Kolleginnen/Kollegen und Vorgesetzten. Im weiteren Projektverlauf hatte Tim jedoch mit vielerlei bürokratischen Hürden zu kämpfen, durch die er sich mutig schlug:

„Ende August gab es ein Gespräch zwischen Tim und der Organisation $^{4}$, Ende September dazu eine E-Mail von der Organisation, die beschreibt, dass für das Projekt eine Betriebserlaubnis für einen offenen Kindertreff notwendig ist, wofür wiederum hohe Anforderungen bestehen. Die avisierte Kooperation mit der Organisation erscheint damit hinfällig. Das Bürgerprojekt kann in der ursprünglichen Form aufgrund zu hoher behördlicher/bürokratischer Auflagen (exemplarisch: Jugendamt [...]) nicht im Ehrenamt umgesetzt werden“" (Auszug aus dem Feldtagebuch, 17.10.2017).

Letztendlich wurde mit der Unterstützung des Vor-OrtBüros eine alternative Herangehensweise gewählt, in der der Ort und die Qualifikation der Betreuungspersonen durch das Vertrauen der Eltern beurteilt wurden. Es kam zu einem Speed-Dating-Termin mit Eltern, Kindern und älteren Personen, die gerne ein Kind außerhalb der Kindergartenzeiten betreuen wollten. Diese Veranstaltung hatte einen großen Zulauf und es konnten dadurch einige ehrenamtliche Großeltern vermittelt werden. Schließlich musste Tim jedoch aufgrund persönlicher Gründe und wegen des immens hohen, bürokratischen Aufwands beim Mittelabruf im Kleinprojektefonds und den damit verbundenen personellen Verantwortlichkei-

4 Der Name der Organisation wurde durch „Organisation" ersetzt. ten in der Stadtverwaltung selbst das Bürgerprojekt nach etwas über einem Jahr Laufzeit aufgeben. Der bürokratische Aufwand hatte zu viel Zeit in Anspruch genommen, was für einen Einzelkämpfer nicht mehr leistbar war. Tim schätzt, dass 50-60 \% seiner Zeit als Engagierter nur für buchhalterische und organisatorische Tätigkeiten aufgebracht wurden. Das hat inn extrem demotiviert.

Auch Ben gab sein Bürgerprojekt nach eineinhalb Jahren auf, nachdem bestimmte Projektverläufe nicht zufriedenstellend gelaufen waren und seine ursprüngliche Idee, die Verbindung zwischen Kultur und Sport, gar nicht zum Tragen gekommen war. Letzten Endes waren es persönliche und berufliche Gründe, die zum Abbruch des Projektes führten, denn Ben verließ anschließend Weißwasser. Zu Bens Bürgerprojekt gab es eine Neuauflage: Ein Jugendlicher, der sich zu der Zeit gerade im Praktikum bei einem soziokulturellen Verein und kurz vor Aufnahme seines Studiums befand, legte ein Kunstprojekt auf, das nun jährlich einmal mit wechselnden Protagonisten stattfinden soll und dann vom Verein selbst getragen wird.

\subsubsection{Engagement als Jobgenerator}

Dass Engagement in strukturschwachen Räumen genutzt wird, um bessere Chancen auf dem Arbeitsmarkt zu haben, wurde bereits in Kapitel 2.2 erläutert. Im Projekt „Kleinstadt_gestalten“ waren es sechs junge männliche und weibliche Geflüchtete mit ihren Familien, die durch ihr Bürgerprojekt zum einen den interkulturellen Austausch fördern, zum anderen in Weißwasser ,ankommen' wollten. Durch gastronomische Angebote wollten sie sich einen Lebensunterhalt verdienen, so war zumindest der Plan zu Anfang des Projektes. Auch Lukas, ein arbeitsloser Alleinerziehender, verband sein Engagement in einem anderen Bürgerprojekt mit der Jobsuche:

\begin{abstract}
„Wenn man selber in einer Situation ist, wo für einen selber die berufliche Perspektive sehr unklar ist, dann ist es eigentlich ganz normal, dass man einen großen Teil der Energie dafür verwenden muss. Auch sagen wir mal die Existenzsicherung, ein Kind zu haben, da ist das Engagement eben das Werkzeug dazu" (Lukas, 01.11.2017).
\end{abstract}

Die Geflüchteten wurden von Hans unterstützt, der hauptamtlich mit seinem Verein soziale Projekte fördert. Im weiteren Verlauf des Projektes gestaltete es sich zunehmend schwieriger, einen anderen ehrenamtlichen Koordinator zu finden, der Hans ablösen sollte, damit dieser sich seinen eigenen hauptamtlichen Projekten widmen konnte. Ein weiterer schwieriger Faktor war die Sprachbarriere der Geflüchteten. Deshalb sollte der 
Koordinator die Aktionen, die eigentlich in Kooperation mit der lokalen Gastronomie stattfinden sollten, in ihrer Anlaufzeit unterstützend begleiten. Diese Aufgabe war jedoch mit viel kommunikativer und organisatorischer Arbeit verbunden. Hans fasst diese komplexe Aufgabe in der Aussage zusammen:

„Um dieses Projekt weiter zu führen, braucht man Leute, die es sich zutrauen, Fremde anzusprechen und sozusagen mitzunehmen" (Hans, 23.01.2019).

Zudem hegten die Vertreterinnen und Vertreter der örtlichen Gastronomie Bedenken gegenüber dem kulinarischen Angebot, der Gesundheit und Hygiene. Sie brachten zum Ausdruck, dass grundsätzlich kein Interesse an einem interkulturellen Abend bestünde. Äußerungen aus der Stadtgesellschaft bestätigten dies. Zusätzlich wurde der Kleinprojektefonds als zu bürokratisch und unübersichtlich wahrgenommen. Deshalb wurden kaum Mittel abgerufen, stattdessen eine Spendendose aufgestellt und die Ehrenamtspauschale in Anspruch genommen:

\begin{abstract}
"... da müssten sich entweder Fördermittelgeber oder die Stellen, die für die Umsetzung des Programms zuständig sind, die müssten sich drüber im Klaren sein, also wenn wir Ehrenamtsprojekte fördern wollen, dann muss die Inanspruchnahme der Mittel und die Bewirtschaftung so übersichtlich und unkompliziert sein, dass dort nicht die Leute, die das ehrenamtlich machen, die Hälfte ihrer Zeit darauf verwenden, dort irgendwelche Vorgänge zu eruieren, die die Behörden brauchen oder die Fördermittelstelle braucht, weil dann macht man's tot. Also dann, das geht gar nicht. Das ist keine Ehrenamtsförderung, das ist Ehrenamtsbehinderung dann letztendlich"(Hans, 23.01.2019).
\end{abstract}

Auch die Unterstützung vom Vor-Ort-Büro nahm Hans kaum in Anspruch, da er sich mit Projektmanagement schon von Berufs wegen auskannte. Unterstützung für das Bürgerprojekt bekam er seit der Hälfte der Projektlaufzeit von den Bundesfreiwilligen. Damit das Bürgerprojekt auch ohne die lokale Gastronomie weiterlaufen kann, werden auch künftig die kulinarischen Leckereien auf städtischen Festen und Veranstaltungen angeboten. Mittlerweile werden sie sogar vermehrt für öffentliche Veranstaltungen nachgefragt. Das Bürgerprojekt blieb und bleibt damit auch weiterhin der Stadt erhalten.

\subsubsection{Engagement und Eigeninteressen}

Im Engagement werden auch Eigeninteressen verfolgt, die letztendlich dem Gemeinwohl zugutekommen, da es allgemeine Bedarfe anspricht, wie im Falle der fünf Schü- lerinnen und Schüler im Alter zwischen 15 und 18 Jahren, die zu Projektbeginn zum Großteil auf das örtliche Gymnasium gingen. Vor allem Felix und Lisa lag ein Projekt am Herzen: Sie wollten einen Jugendtreff etablieren aus dem Bedarf heraus, dass es für sie, ihre Freunde und Klassenkameradinnen/-kameraden keinen Treffpunkt gab, in dem sie sich in ihrer Freizeit mit anderen treffen konnten, in dem Konzerte, Veranstaltungen oder Workshops angeboten wurden, wo jeder gern gesehen war:

\begin{abstract}
„... der Abend ist ein Problem und damit fing das dann an. Was stellen wir uns vor? Was fehlt in Weißwasser? Eben dieser Treff, und dadurch ging das dann nach und nach, mit den Mädels noch Ideen gesammelt. Dann waren auch noch andere da, auch Jugendliche, weiter Ideen gesammelt und dann Kontakte ausgetauscht, Adressen“ (Felix, 31.01.2017).
\end{abstract}

Die Werbung auf Facebook und die Guerilla-Werbung in der Stadt lockten sie zur Auftaktveranstaltung des Forschungsprojektes, wo die Ideen für die zukünftigen Bürgerprojekte gesammelt und Einladungen zur Summer School ausgesprochen wurden, um diese Ideen weiterzuentwickeln, sodass aus Ideen ein Engagement mit Aktionen und Veranstaltungen entstehen konnte. Um ihr Bürgerprojekt in die Tat umzusetzen, brauchten sie Räumlichkeiten und einen Träger, der die Funktion der Betreuung bei Jugendlichen unter 18 Jahren übernehmen konnte. Um beide wesentlichen Rahmenbedingungen zu erfüllen, gab es ein zähes Ringen, da es von mehreren Seiten Ansprüche gab, zum Beispiel Betreuungsansprüche von einem anderen lokalen Trägerverein (Konkurrenz zwischen den Trägervereinen) und hohe Ansprüche an die Räumlichkeiten von den Jugendlichen selbst, die nicht aus dem Weg zu räumen waren. Die Stadtverwaltung unterstützte die Suche nach den Räumlichkeiten oder nach Betreuung nicht. Dazu merkt Leon von einem anderen Bürgerprojekt an:

„Die Jugendlichen brauchen ein Erfolgserlebnis, um am Ball zu bleiben, und Unterstützung dazu“ (Leon, 27.09.2018).

Obwohl sie im Rahmen von „Kleinstadt_gestalten“ bei ihrer Suche nach Räumlichkeiten und organisatorischen Lösungen stark vom Vor-Ort Büro unterstützt wurden, entschieden sich die Jugendlichen, das Bürgerprojekt nach fünf Monaten erfolgloser Suche aufzugeben, da die weitere Motivation fehlte. Drei Protagonisten waren bereits vorher abgesprungen. Felix stand vor dem Abitur und der anschließenden Ausbildung, Lisa hatte sich für ein Austauschjahr im Ausland entschieden.

Lukas hatte mit seinem Mitstreiter schon vor dem Projektstart von „Kleinstadt_gestalten“ eine Projektidee 
und auch schon drei Aktionen mit Unterstützung des Bürgermeisters zur Müllbeseitigung in der Stadt gestartet. Er wollte erreichen, dass die Menschen nicht nur meckern, sondern selbst mit anpacken, dass die Bürgerinnen und Bürger ein Bewusstsein entwickeln, selbst tätig zu werden und teilzuhaben an der Mitgestaltung ihrer Stadt. Lukas ist Zugezogener mit einem Hauptschulabschluss und einer Ausbildung. Zum Projektstart war der alleinerziehende Vater Hausmann. Vom Projekt erhoffte er sich eine breite Unterstützung, um sein Vorhaben breiter aufzustellen und auch Räumlichkeiten für seine Geräte, für Aktionen und Publikumsverkehr zu finden. Mit seinem Bürgerprojekt wollte er eine saubere Stadt mit bespielbaren, sicheren Spielplätzen schaffen.

\begin{abstract}
„Naja, wenn sich mehr Leute anschließen und sich gegebenenfalls daraus eine richtige Organisation entwickelt, sage ich mal, ne. Die halt, sage ich mal, auch angesehen ist, auch nicht nur von den Bürgern, sondern auch von den Firmen" (Lukas, 31.01.2017).
\end{abstract}

Der Verlauf seines Projektes war geprägt von vielen Hürden, angefangen damit, dass eine Projektkalkulation verlangt wurde, die inm viel abverlangte, dass seine Mitstreiterinnen/Mitstreiter wechselten, der Kleinprojektefonds viel zu bürokratisch und nicht engagementfreundlich zu handhaben war. Zudem gab es für manche Maßnahmen keine klaren Regeln, was zur Folge hatte, dass er durch fehlende Kommunikation ausgebremst und demotiviert wurde. Aber auch persönliche Umstände führten streckenweise zur Behinderung seines Bürgerprojektes. Dazu trug zum Teil auch die hierarchische, starre Projektstruktur von „Kleinstadt_gestalten“ bei, die kaum Flexibilität zuließ, da auf Vorschriften beharrt wurde, die sich von Engagierten aufgrund von Zeitmangel oder Mangel an Qualifikation kaum beachten ließen. Zudem wurden den Engagierten keine Leitlinien zum Abruf des Kleinprojektefonds an die Hand gegeben, was zu viel Verwirrung, Frust und wenig Mittelabfluss führte.

\footnotetext{
„Ich stelle halt immer wieder im Rahmen des Projektes fest, dass in dem Projekt selber irgendwo so ein Stück weit die Struktur fehlt. Also, es gab da nie klare Regeln, die irgendwo schriftlich festgehalten werden. Wer darf was oder sonst was. Wie ich das meinte mit der Handkasse. Zum Anfang hat man uns gesagt, 400 Euro, ab 400 Euro müssen wir drei Angebote einholen. Und dann waren es auf einmal 100 Euro, wo ich sage, so was muss von vornherein geklärt werden" (Lukas, 23.01.2019).
}

Lukas vernetzte sich stark mit anderen Aktiven und Vereinen in der Stadt und bekam starke Unterstützung vom Vor-Ort-Büro sowie Räumlichkeiten für das Bürger- projekt. Acht Monate vor Projektende von „Kleinstadt_ gestalten" bekam er einen Job im Schichtdienst mit 30 Arbeitsstunden in der Woche. Bis zum Projektende war noch nicht klar, wer die Raummiete und die Aktionen des Bürgerprojektes weiter finanzieren sollte. Zudem fiel sein Mitstreiter auf unbestimmte Zeit aus. Damit war unklar, ob das Projekt, wie von Lukas intendiert, weiter betrieben werden konnte.

\subsubsection{Engagement und Gemeinwohl}

Drei junge Frauen verbanden mit ihren Bürgerprojekten vornehmlich Gemeinwohlinteressen, wobei alle drei eine ähnliche Bedarfsorientierung verfolgten: Austausch und Vernetzung. Jana ist Rückkehrerin, Mutter dreier Kinder, hat studiert und arbeitet halbtags. Den Anreiz, ihr Bürgerprojekt auf den Weg zu bringen, drückt sie wie folgt aus:

\begin{abstract}
„Naja, zu bestimmten gesellschaftlichen Fragestellungen, denke ich, findet man auch nur Antworten, wenn man im Austausch mit anderen ist. [...] Was haben andere für Gedanken dazu, für Ideen dazu, und nur daran kann ich mich eigentlich weiterentwickeln, denke ich. Was dann, hoffe ich, auch aus so einer Gruppe heraus auch wieder in die Gesellschaft oder eben in die Stadt hineinwirkt. Also, man geht ja dann doch wieder raus mit neuen Gedanken, mit neuen Blickrichtungen und, ja, in irgendeiner Weise wirkt es auf die Stadt zurück, und ich hoffe auf positive Weise eben“"(Jana, 31.01.2017).
\end{abstract}

Sie wollte mit ihrem Projekt den Ideenaustausch und die Vernetzung von Menschen fördern und so die Kräfte zur Weiterentwicklung der Stadt bündeln. In ihrem Engagement wurde sie vor allem durch ihren Ehemann unterstützt und nur gelegentlich vom Vor-Ort-Büro, da sie sich durch ihre Arbeit im Projektmanagement auskannte.

Ein weiteres Bürgerprojekt hat Marie auf den Weg gebracht, um den Informationsaustausch in der Stadt zu fördern. Sie hat ihr Fachabitur und eine Ausbildung im Dienstleistungsbereich abgeschlossen. Täglich pendelte sie eine Stunde zu inrem Bürojob mit einer Arbeitszeit von 30 Stunden pro Woche und hatte noch keine Familie gegründet. Anna wollte mit ihrem Bürgerprojekt den kulturellen Austausch in der Stadt fördern und bezog dazu auch die Diaspora ${ }^{5}$ in ihre Treffen mit ein. Sie hat studiert, ist zusammen mit ihrem Partner ins Umland von

5 Mit „Diaspora“ sind ehemalige Bewohnerinnen und Bewohner von Weißwasser gemeint, die in anderen Teilen Deutschlands und der Welt leben, sich aber immer noch für ihre Heimatstadt Weißwasser interessieren und sie regelmäßig besuchen. 
Weißwasser zurückgekehrt und arbeitet nun als Selbstständige. Auch sie hatte noch keine eigene Familie gegründet. Ihr Projekt war ein Nachrücker, das nur acht Monate vor Projektende Zeit hatte, sich zu etablieren. Sie hatte dabei starke Unterstützung von einem Projektmitarbeiter und Freunden, die ihr halfen, für ihre Aktionen in der Stadt und auf Facebook zu werben und sie auch während der Aktionen unterstützten.

Die drei Projektverläufe gestalteten sich sehr unterschiedlich: Jana zeigte sich zum Start ihres Bürgerprojektes sehr euphorisch und organisierte ihre Treffen zum Ideenaustausch mit großem Eifer. Im weiteren Verlauf jedoch fehlte ihr immer mehr die Zeit, um weitere Treffen zu organisieren und eine Kontinuität in ihr Projekt hineinzubringen. Gründe für den entstandenen Zeitmangel waren zum einen, dass sie stärker in ihre Arbeit eingebunden wurde, ihre Arbeitsstunden wurden heraufgesetzt, zum anderen spielten familiäre Gründe eine Rolle. Selbst die Suche und Aktivierung eines Mitstreiters zur Verstetigung ihres Projektes gestaltete sich angesichts des akuten Zeitmangels sehr schwierig. Jana konnte selbst die angebotene Unterstützung des Vor-Ort-Büros und ihres potenziellen Mitstreiters kaum nutzen. Jana merkt dazu an:

„Kontakte pflegen, ja, das ist was, was ich jetzt schon auch so ein Stück angebahnt hab' dadurch, dass ich einfach viel zu wenig Zeit hab' für die Ideen, die ich so hatte und habe, hat sich das so ergeben, dass ein paar andere Leute da entweder mit eingestiegen sind oder auch mit einer ähnlichen Idee kamen, irgendwie da jetzt gemeinsame Sache machen oder die selber auch was starten. Ja, also das ist für mich auf alle Fälle ein Punkt, ja, weil ich das alleine nicht schaffe" (Jana, 04.10.2018).

Dennoch gelang es, das Projekt zu verstetigen, indem Jana ihr Bürgerprojekt an ein ebenfalls verstetigtes Bürgerprojekt aus „Kleinstadt_gestalten“ angedockt hat und in dessen Rahmen weiterhin ihre Treffen stattfinden lassen konnte.

An Verstetigung war bei Marie erst einmal kaum zu denken, gestaltete sich doch der ganze Verlauf des Projektes schwierig. Zudem brachte sie keinerlei Projektmanagementerfahrung mit und fühlte sich streckenweise allein gelassen, da durch einen Projektmitarbeiter, der sie besonders unterstützen wollte, kaum Informationen zu ihr vordrangen. Außerdem bestanden unterschiedliche Vorstellungen zwischen ihr und dem Projektmitarbeiter über die Ziele des Bürgerprojektes, wie Informationen zu Veranstaltungen, Vereinen und Ähnliches an die Bürger herangetragen werden sollten. Einige Entscheidungen über den weiteren Verlauf des Bürgerprojektes fällte der Projektmitarbeiter im Alleingang. Marie fühlte sich dadurch manchmal ihres eigenen Bürgerprojektes beraubt. Selbst als sie eine Mitstreiterin für inr Projekt gewinnen konnte, fehlte ihr die Motivation und die Zeit, das Projekt fortzusetzen. Da sie ihre Arbeitsstelle gewechselt hatte, nun Vollzeit arbeitete und täglich zwei Stunden pendeln musste, blieb kaum noch Zeit, das Bürgerprojekt weiter zu verfolgen. Ihre Mitstreiterin Ella, eine nach Weißwasser Zugezogene, übernahm das Projekt. Marie fasst ihren Entschluss, das Bürgerprojekt abzugeben, so zusammen:

\begin{abstract}
„Also, das war ja so, dass ich relativ zeitig schon so ein bisschen gezweifelt hab; weil ich mir das ja ein bisschen anders vorgestellt habe. Und hab' dann aber trotzdem weitergemacht. Hatte die Zweifel immer so im Hintergrund, na ja, und irgendwann hab'ich dann auch eben durch den neuen Job, und da ich ja auch dann Vollzeit gehe mit dem Arbeitsweg, halt gemerkt, das funktioniert nicht. Und dieser Gegensatz, weniger Zeit, aber nicht mehr Unterstützung, dann eben gesagt, okay, das funktioniert einfach nicht. Ich hab“ ja auch noch für mich ein Privatleben. Und ich hatte manchmal auch das Gefühl, die Ergebnisse, die ich dann doch gebracht habe, sind dann irgendwie im Sande verlaufen. [...], jetzt hast Du Dir Deine wenige Zeit genommen, und irgendwie ist da nie das rausgekommen, was ich mir vorgestellt habe. Und das war nicht bloß ein, zwei Mal so, das war dann halt mehrmals so. Und dann wollte ich nicht, dass es so weitergeht, und hab dann das eben beendet" (Marie, 25.07.2017).
\end{abstract}

Als Ella das Projekt von Marie übernahm, war sie vor Ort in Vollzeit beschäftigt und hatte noch keine eigene Familie gegründet. Unterdessen hatte auch Ellas Arbeitgeber Gefallen an ihrem Bürgerprojekt gefunden und es für betriebliche Zwecke vereinnahmt, was es schwierig gestaltete, die ehrenamtliche Ausrichtung des Bürgerprojektes aufrechtzuhalten. Es fanden sogar Projekttreffen statt, über die sie nicht informiert wurde. Ella war kurz davor, das Projekt fallen zu lassen, denn sie fand sich nicht mehr in dem Projekt wieder, war stark frustriert und demotiviert:

„Aufgrund eines generellen, von ihr wahrgenommenen Informationsdefizits stellte sich in der Vergangenheit ein Gefühl ein, außen vorgelassen zu werden. Zum Teil führte dies zum Verlust von Motivation [...]" (Auszug Feldtagebucheintrag 10.04.2018). „Mit der Entwicklung des Projektes bis zum jetzigen Zeitpunkt findet Ella die ursprüngliche Idee, die sie unterstützen wollte, kaum mehr wieder (Anspruch vs. Wirklichkeit). Ihre eigens gewählten Aufgaben sind obsolet auf Eis gelegt, und sie bekleidet eine Rolle, die sie nicht selbst gewählt hat. Was ihre Kapazitäten angeht, wird sie nicht viel mehr leisten können und wollen, [...]" (Auszug Feldtagebucheintrag 06.06.2018). 
Letztendlich wurden vom Arbeitgeber und vom Projektkoordinator doch noch Lösungen aufgezeigt, die dem Projekt wieder seinen freiwilligen Charakter gaben und ein sehr erfolgreiches Bürgerprojekt in Zusammenarbeit mit einem Unternehmen entstehen ließen, da es eine große Zahl an Bürgerinnen und Bürgern in Weißwasser mit aktuellen Informationen versorgt. Ella fasst ihren Teil des Projektes sehr treffend zusammen:

„Ja, also ich find', das letzte Mal, wo ich hier gesessen hab“,
hab“ ich eigentlich mehr oder weniger kundgetan, dass das
Projekt irgendwie für mich zu Ende ist an der Stelle. Gott sei
Dank gab es wirklich nachher nochmal Gespräche, also, ich
hab' nochmal das Gespräch bei uns im Haus gesucht. Da hat
sich nämlich bei einer Position da irgendwas geändert gehabt,
das hat jetzt eine Kollegin übernommen sozusagen, [...] Und
dann haben wir nochmal das gemeinsame Gespräch mit dem
Projektkoordinator zusammen geführt, und haben nochmal
geguckt, [...] wie können wir denn die Aufgabe irgendwie auf-
teilen. [...] Und jetzt ist es aktuell so, dass es, bis Ende der
Woche läuft die erste Testphase, ich hab'auch selber mal rein-
geguckt, und ich hab“ es offenbar geschafft"(Ella, 04.10.2018).

Auch Anna hat es geschafft, inr Bürgerprojekt zu verstetigen, denn sie erfuhr, wie oben bereits erwähnt, massive Unterstützung von einem Projektmitarbeiter aus dem Vor-Ort-Büro, der ihr auch alles Bürokratische von „Kleinstadt_gestalten“ abnahm. Ihr Format, eine Plattform für kulturellen Austausch anzubieten und dies mit Livemusik, Vorstellungen und Kulinarik zu verbinden, wurde sehr gut angenommen von den Bürgerinnen und Bürgern in Weißwasser, aber auch von jenen, die ihren Lebensmittelpunkt in einer anderen Stadt gefunden haben. Anna wurde auch nach dem Ende von „Kleinstadt_gestalten" von einem soziokulturellen Verein mit Räumlichkeiten und Equipment unterstützt, das es ihr ermöglichte, ihr Bürgerprojekt zu verstetigen.

Überblickt man das Projekt „Kleinstadt_gestalten“ als Ganzes im Verlauf, sind vor allem starre Strukturen im Projekt, das Denken in Hierarchien und Bürokratien, wechselnde Verantwortlichkeiten, Vereinnahmung, unklare, missverständliche oder fehlende Kommunikation und auf städtischer Seite mangelnde personelle Unterstützung und damit verbunden Zeitmangel als wesentliche Hürden beim Verlauf der Bürgerprojekte zu nennen, die zu Behinderungen bis hin zum Abbruch geführt haben. Als Push-Faktoren und klare Förderungen der Bürgerprojekte anzuerkennen sind, dass sie begleitet wurden durch die Wertschätzung von Freiwilligenarbeit, durch den Rückhalt und die Unterstützung von Familie, Kolleginnen/Kollegen, Freundinnen/ Freunden und Vor-Ort-Mitarbeiterinnen/-Mitarbeitern. Weiterhin waren organisierte gemeinsame Treffen und gegenseitiger Austausch maßgeblich für den Erfolg der Bürgerprojekte. Verstetigen konnten sich die Einzelkämpferprojekte durch die Kooperation und Vernetzung mit anderen Organisationen und Unternehmen in Weißwasser. Insofern greift die kleinstädtische Ankerfunktion, weil sie damit einige wenige Möglichkeiten zur Verstetigung bietet.

\section{Diskussion: Eigensinn trifft Gemeinsinn}

Betrachtet man die in Kapitel 2 vorgestellten fünf Dimensionen des zivilgesellschaftlichen Engagements vor dem Hintergrund der empirischen Befunde, so gewinnen einige Dimensionen stärker an Bedeutung, andere erscheinen wiederum weniger wichtig und sind gerade im Kontext der geschrumpften, strukturschwachen Kleinstadt neu zu bewerten. Für diese Neubewertung lassen sich aus den vier Anreizoptionen Einzelfaktoren ableiten, die maßgeblich ihr Engagement beeinflussen (vgl. Abbildung 1).

Neu einzuordnen ist die Dimension, wonach zivilgesellschaftliches Engagement nicht das Ziel verfolgt, berufliche Qualifikationen zu erwerben oder weiter zu entwickeln. Simonson, Ziegelmann, Vogel et al. (2016: 19) merken dazu an, dass im Freiwilligensurvey die Motivation, mit Engagement Qualifikationen zu erwerben, seltener ein Wunsch war. Tatsächlich gewinnt in einer sozioökonomisch schwachen Stadt wie Weißwasser der Einzelfaktor „berufliche Qualifikation“ jedoch stark an Gewicht, bietet er doch eine Möglichkeit zur Weiterbildung oder Qualifikation für den Arbeitsmarkt, wie auch die Option, berufliche und ehrenamtliche Netzwerke gleichermaßen zu nutzen. Damit bestätigt sich auch im Projekt „Kleinstadt_gestalten“ der Befund von Olk und Gensicke (2014: 178, 203), dass zivilgesellschaftliches Engagement unter Schrumpfungsbedingungen eine andere Sinnzuweisung erfährt. Die Engagierten gewinnen ihr Selbstbewusstsein zurück und wollen ihr Wissen dem Arbeitsmarkt zur Verfügung stellen. Die Dimension, dass durch zivilgesellschaftliches Engagement keine direkte politische Macht ausgeübt wird, trifft auch auf den Kontext Weißwassers zu. Der Einzelfaktor Bewusstseinsbildung bildet eine neue Dimension von zivilgesellschaftlichem Engagement, denn vor allem Lukas hat sich mit seinem Bürgerprojekt das Ziel gesetzt, Bewusstseinsbildung für die Teilhabe der Bürgerinnen und Bürger an der Mitgestaltung der Stadt zu betreiben und basisdemokratische Prozesse in Gang zu bringen. 

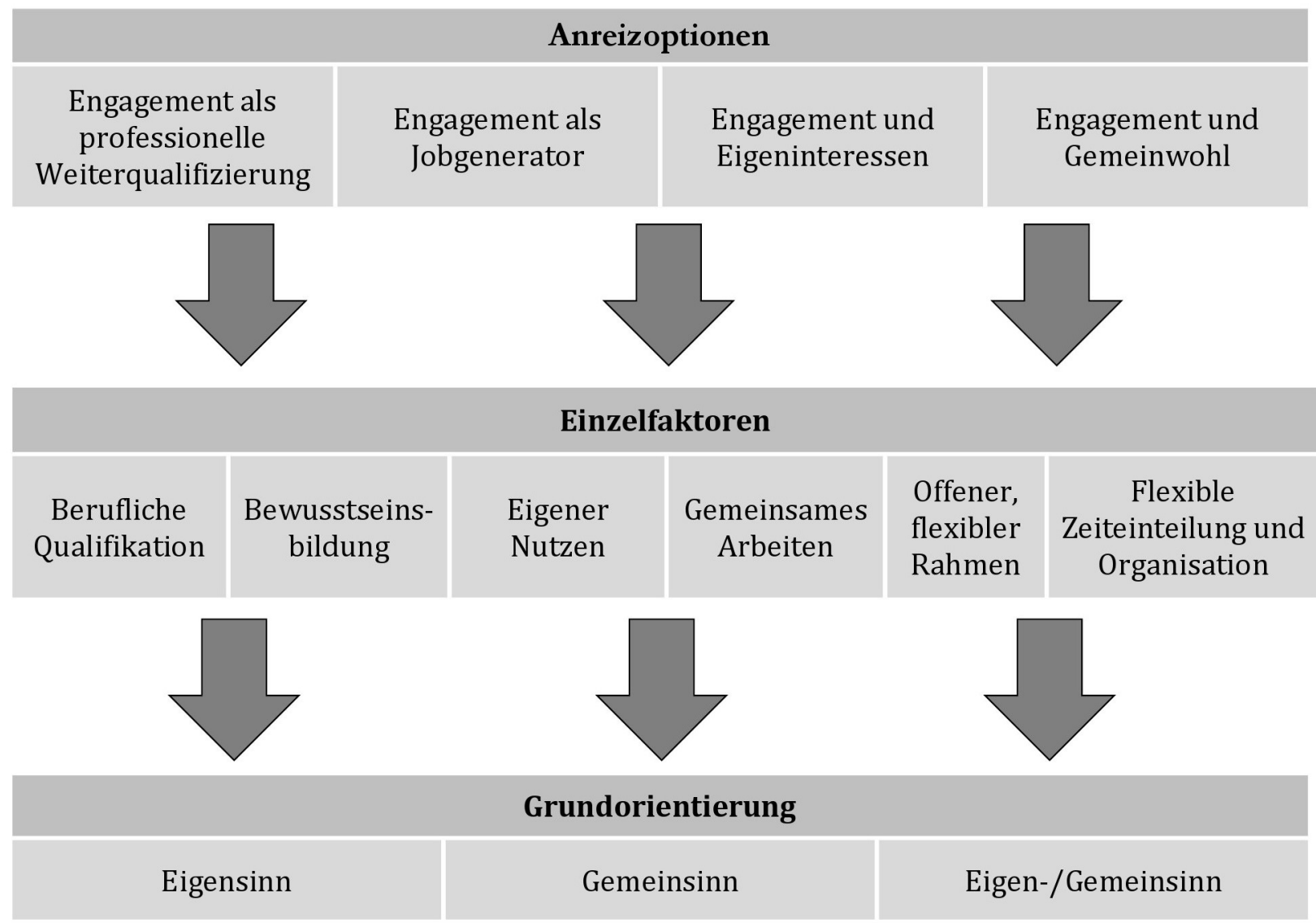

Abbildung 1: Anreizoptionen und Einzelfaktoren des Engagements und Grundorientierung Motivation

Gemeinwohlorientierte Arbeit als dritte Dimension zivilgesellschaftlichen Engagements ist zumindest vielschichtiger zu denken. Ihr steht der Einzelfaktor „eigener Nutzen" gegenüber. Dieser wurde vor allem bei den jungen Männern und Jugendlichen häufig als erster Anreiz genannt, um Bürgerprojekte auf den Weg zu bringen. Hier gewinnt auch Nadlers (2017: 507) Hinweis an Gewicht, auf die Eigeninteressen der Engagierten stärker einzugehen. Im Falle der Bürgerprojekte war dies, einen Jugendtreff für sich und ihre peer group zu etablieren. Nicht zuletzt spielen auch die Aneignung von Qualifikationen und Jobgenerierung mit in diesen Einzelfaktor hinein. Auch Kelemen, Mangan und Moffat (2017: 1252) beschreiben diesen Einzelfaktor und merken dazu an: "While enjoying and deriving pleasure and satisfaction from volunteer work, many of the volunteers see clear individual benefits arising from this unpaid work, be they in terms of personal satisfaction, increase in confidence, learning new skills, getting paid employment and meeting new people."

Angesichts der städtischen und organisatorischen Rahmenbedingungen gewinnen zwei Dimensionen zivil- gesellschaftlichen Engagements stärker an Gewicht. Zum einen ist es die Autonomie des Engagements, die auch impliziert, dass eigene Ideen eingebracht werden können. Aufgrund des hierarchischen, bürokratischen Projektcharakters mit verlaufsweise unklarer Struktur kam diese Dimension bei „Kleinstadt_gestalten“ nur ungenügend zum Tragen. Dies bestätigt die Forderung von Kummel und Nadler (2018: 59), bürokratische Hürden im freiwilligen Engagement abzubauen. Gerade angesichts einer fehlenden kritischen Masse an potenziell Engagierten in einer geschrumpften Kleinstadt, kann das autonome, selbstbestimmte Engagement eine Chance auf Mitgestaltung der eigenen Kleinstadt sein. Der Einzelfaktor "offener, flexibler Rahmen“ bezieht sich auf die Art der Projektsteuerung. Dieser Einzelfaktor gewinnt vor allem an Notwendigkeit, wenn Interessenkonflikte und Vereinnahmung von Projektkoordinator und Mitarbeiter das Engagement der vornehmlich weiblichen Protagonisten in „Kleinstadt_gestalten“ ausbremsen oder gar stoppen, wie im Fall von Marie und Ella.

Zum anderen ist es die Dimension der Einbettung zivilgesellschaftlichen Engagements im öffentlichen 
Raum und in zivilgesellschaftliche Organisationen, die aufgrund des Einzelkämpfertums der Engagierten stärkeres Gewicht bekommt. Zur Verstetigung der Bürgerprojekte war deren Andocken an eine andere Organisation ein wichtiger Push-Faktor. Deshalb ist der Einzelfaktor "gemeinsames Arbeiten" für das Bündeln des Engagementpotenzials essenziell wichtig. Nicht zuletzt bedient gemeinsames Arbeiten auch Eigeninteressen, wenn laut Kelemen, Mangan und Moffat (2017: 1252) vor allem Spaß, eigene Zufriedenheit und neue Kontakte einen Anreiz bilden. Ein weiterer Einzelfaktor verdeutlicht, dass diese Dimension ebenfalls vielschichtiger gedacht werden muss, denn der akute Zeitmangel, den gerade die jungen, engagierten Frauen im Verlauf ihres Projektes beschrieben haben, verdeutlicht den Einfluss des Einzelfaktors „flexible Zeiteinteilung und Organisation“. Es gibt häufig Lebensphasen, in denen für freiwilliges Engagement keine Zeit bleibt, weshalb sich die Protagonisten nur hin und wieder einbringen können. Ehrhardt (2011: 215) und Hollstein (2015: 294) nennen dieses Phänomen „perforierte Engagementverläufe“. Eine zeitliche Flexibilität in einem eng getakteten Förderprojekt ist jedoch kaum zu gewährleisten. Vielmehr fordert zeitliche und organisatorische Flexibilität Kontinuität in der Engagementförderung. Dies bestätigt auch die Forderungen von Steinführer, Küpper und Tautz (2012: 5) und der Enquete-Kommission des Landtags Brandenburg (2018: 85-86) nach mehr Unterstützung und dadurch nach mehr Verlässlichkeit und Kontinuität im Engagement. Dies gilt gerade für eine Kleinstadt, die auf freiwilliges Engagement angewiesen ist, um Bereiche der Daseinsvorsorge abzudecken.

"Die Motive der Engagierten sind vielfältig" (Simonson/Ziegelmann/Vogel et al. 2016: 19). Im Freiwilligensurvey 2014 lauteten die Hauptaussagen zur Motivation, im Engagement sei das Zusammenkommen mit anderen Menschen und um die Gesellschaft mitzugestalten ausschlaggebend (Simonson/Ziegelmann/Vogel et al. 2016: 19), welche sich durchaus mit Befunden im Projekt „Kleinstadt_gestalten“ deckt. Die Motivation, sich zivilgesellschaftlich zu engagieren, kann im Falle von „Kleinstadt_gestalten“ auf drei Grundorientierungen zurückgeführt werden: Eigensinn, Gemeinsinn und eine Mischform aus beiden (vgl. Abbildung 1). Auch Steffen und Fothergill (2009: 42-43) und, darauf aufbauend, Kelemen, Mangan und Moffat (2017: 1253) vertreten die Position, dass Engagement sowohl individuelle wie gemeinschaftliche Motivationen berücksichtige und es den Engagierten ermögliche, sich für das Gemeinwohl zu engagieren und gleichzeitig eine persönliche Befriedigung aus dem kollektiven Handeln zu ziehen. Wenn die Grundorientierung der Motivation auf Eigensinn beruht, dann stehen in erster Linie eigene Interessen und eigener Nutzen beim Engagement im Vordergrund. In der Umsetzung des Engagements zeigt sich dann der gemeinwohlorientierte Charakter.

Denkt man die Mischform aus Eigen- und Gemeinsinn als ein Überlebens- und Schrumpfungsarrangement, wie es von Willisch (2011: 83) beschrieben wurde, so vereinen die Engagierten in effizienter Art und Weise vor allem das Nützliche für sich und die Bedarfe der anderen, um knappe Ressourcen zu bündeln und die Kleinstadtgesellschaft Weißwassers durch ihr Engagement partizipieren zu lassen. Auch die von Küpper, Kundolf, Mettenberger et al. (2018: 231) vorgeschlagenen Bottom-up-Partnerschaften gehören zu den Arrangements, um Schrumpfung zu begegnen und Engagementpotenziale zu bündeln sowie bestimmte zivilgesellschaftliche Gruppen zu aktivieren. Im Falle Weißwassers kann das zivilgesellschaftliche Engagement als stadtgesellschaftlicher wie auch als sozioökonomischer Impulsgeber agieren, wenn bestimmte Rahmenbedingungen erfüllt sind. Die Engagierten werden zu den von Steinführer und Moser (2016: 273) beschriebenen „Leitfiguren“ oder Schlüsselpersonen für das Mitgestalten und Wiederbeleben ihrer Stadt in Bezug auf Informationsaustausch, Netzwerkarbeit, Kulturarbeit und Teilhabe an der Mitgestaltung der Stadt.

Die Verstetigung von Bottom-up-Partnerschaften funktioniert im Falle von Weißwasser nur bedingt mit dem Partner Stadtverwaltung. Infolge prekärer Rahmenbedingungen (Personalmangel und finanzielle Unterversorgung der Stadt) und des dadurch bedingten Wegfalls öffentlicher Dienstleistungen kann dieser kaum ausreichend Unterstützung bieten (vgl. Olk/Gensicke 2014). Dennoch wird in Weißwasser eine „Unterstützungskultur" benötigt, wie sie Gundert (2010: 115) und der Raumordnungsbericht 2017 (BBSR 2017: 54) vorschlagen, um eine Selbstorganisation von Engagement zu ermöglichen (Krimmer 2015: 5). Diese bieten bereits etablierte zivilgesellschaftliche Partner im Sinne der Bottom-upPartnerschaften. Dies sind gemeinnützige Organisationen, die Räume und Equipment zur Verfügung stellen.

Rückblickend auf die in der Einleitung gestellte Frage, wie zivilgesellschaftliches Engagement in einer stark geschrumpften Kleinstadt wie Weißwasser ermöglicht und wie dabei Eigeninteressen der Engagierten stärker berücksichtigt werden können, lautet die Antwort, dass vor allem flexible Engagementstrukturen und eine autonome Projektsteuerung fördernd sein können. Aber auch die lebensweltlichen Umstände der Engagierten haben entscheidenden Einfluss auf die Engagementver- 
läufe. Hierbei zu nennen sind vor allem in bestimmten Lebensphasen berufliche und familiäre Hintergründe wie auch Neuorientierung und berufliche Qualifikation, die einen akuten Zeitmangel oder sogar den Abbruch des Engagements nach sich ziehen. Möglichkeiten, auf diese lebensweltlichen Umstände einzugehen, liegen in einer ermöglichenden „Unterstützerkultur" (Rehm 2008: 5), der diese „perforierten Lebensläufe“ (Ehrhardt 2011: 215; Hollstein 2015: 294) mit mehr oder weniger oder phasenweise keinem Engagement zulassen. Wichtig sind dabei eine Wertschätzung des freiwillig eingebrachten Zeitbudgets sowie Kontinuität und Zuverlässigkeit in der Unterstützung.

Danksagung: Ich danke den anonymen Gutachtern wie auch den Schriftleitern dieser Zeitschrift für ihre hilfreichen Kommentare und Anmerkungen.

\section{Literatur}

Alscher, M.; Priller, E.; Burkhardt, L. (2018): Zivilgesellschaftliches Engagement. In: Bundeszentrale für politische Bildung; Destatis; Wissenschaftszentrum Berlin für Sozialforschung; Sozio-ökonomisches Panel (Hrsg.): Datenreport 2018. Ein Sozialbericht für die Bundesrepublik Deutschland. Bonn, 373381.

Antonov, N. (2016): Voluntary work in Germany and Norway. A comparative study. Berlin. = Opusculum 95.

Aring, J. (2013): Inverse Frontiers - Selbstverantwortungsräume. In: Faber, K.; Oswalt, P. (Hrsg.): Raumpioniere in ländlichen Regionen - Neue Wege der Daseinsvorsorge. Leipzig, 42-56. = Edition Bauhaus 35 .

ARL - Akademie für Raumforschung und Landesplanung (2016): Daseinsvorsorge und gleichwertige Lebensverhältnisse neu denken. Perspektiven und Handlungsfelder. Hannover. = Positionspapier aus der ARL 108.

BAGFA - Bundesarbeitsgemeinschaft der Freiwilligenagenturen e.V. (2014): Monetarisierung - kein Weg zur Förderung des bürgerschaftlichen Engagements. Ein Positionspapier der Bundesarbeitsgemeinschaft der Freiwilligenagenturen (bagfa) e.V. Potsdam.

Barlösius, E.; Neu, C. (2008): Territoriale Ungleichheit: Eine spezifische Ausprägung räumlicher Untergleichheit. In: Barlösius, E.; Neu, C. (Hrsg.): Peripherisierung - eine neue Form sozialer Ungleichheit? Berlin, 17-23. = Materialien der Berlin-Brandenburgischen Akademie der Wissenschaften 21.

BBSR - Bundesinstitut für Bau-, Stadt- und Raumforschung (2017): Raumordnungsbericht 2017: Daseinsvorsorge sichern. Bonn.

Beetz, S.; Huning, S.; Plieninger, T. (2008): Landscapes of Peripherization in North-Eastern Germany's Countryside: New Challenges for Planning Theory and Practice. In: International Planning Studies 13, 4, 295-310. doi: 10.1080/13563470802518909
Bernt, M. (2009): Partnerships for Demolition: The Governance of Urban Renewal in East Germany's Shrinking Cities. In: International Journal of Urban and Regional Research 33, 3, 754-769. doi: 10.1111/j.1468-2427.2009.00856.x

Bernt, M.; Kabisch, S.; Peter, A. (2005): Auswirkungen von Schrumpfung und Stadtumbau auf die Stadtgesellschaft: Der Fall Weißwasser. In: Berichte zur Deutschen Landeskunde 79, 1, 33-57.

Bischoff, S.; Rahn, E. (2015): Der Flickenteppich. In: Zeckra, C.; Amrhein, U.; Sittler, L.; Bischoff, S. (Hrsg.): Generali Engagementatlas 2015. Köln, 10-16.

BMFSFJ - Bundesministerium für Familie, Senioren, Frauen und Jugend (2012): Erster Engagementbericht 2012. Für eine Kultur der Mitverantwortung. Berlin.

BMFSFJ - Bundesministerium für Familie, Senioren, Frauen und Jugend (2017): Zweiter Bericht über die Entwicklung des bürgerschaftlichen Engagements in der Bundesrepublik Deutschland. Berlin.

Deutscher Bundestag (2002): Bericht der Enquete-Kommission „Zukunft des Bürgerschaftlichen Engagements“. Bürgerschaftliches Engagement: auf dem Weg in eine zukunftsfähige Bürgergesellschaft. Berlin. = Drucksache $14 / 8900$.

Ehrhardt, J. (2011): Ehrenamt: Formen, Dauer und kulturelle Grundlagen des Engagements. Frankfurt am Main.

Flick, U. (2012): Qualitative Sozialforschung: Eine Einführung. Reinbek bei Hamburg.

Gabler, J.; Kollmorgen, R.; Kottwitz, A. (2016): Wer kommt? Wer geht? Wer bleibt? Eine Studie zur Verbesserung der Verbleibchancen qualifizierter Frauen im Landkreis Görlitz. Görlitz.

Gundert, S. (2010): Bürgerschaftliches Engagement im ländlichen Raum. Das Beispiel Biedenkopf-Kombach: Zukunftschance trotz demographischen Wandels? In: Standort - Zeitschrift für Angewandte Geographie 34, 4, 110-115. doi:10.1007/s00548010-0148-7

Hollstein, B. (2015): Ehrenamt verstehen: Eine handlungstheoretische Analyse. Frankfurt am Main.

Kausmann, C.; Simonson, J. (2016): Freiwilliges Engagement in West- und Ostdeutschland sowie den 16 Ländern. In: Simonson, J.; Vogel, C.; Tesch-Römer, C. (Hrsg.): Freiwilliges Engagement in Deutschland. Der Deutsche Freiwilligensurvey 2014. Wiesbaden, 573-600. doi: 10.1007/978-3-658-126445_23

Kelemen, M.; Mangan, A.; Moffat, S. (2017): More than a ,little act of kindness'? Towards a typology of volunteering as unpaid work. In: Sociology 51, 6, 1239-1256. doi: 10.1177/0038038517692512

Klie, T.; Marzluff, S. (2012): Engagement gestaltet ländliche Räume. Chancen und Grenzen bürgerschaftlichen Engagements zur kommunalen Daseinsvorsorge. In: Zeitschrift für Gerontologie und Geriatrie 45, 8, 748-755. doi: 10.1007/s00391-012-0376-5

Krimmer, H. (2015): Einleitung. In: Zeckra, C.; Amrhein, U.; Sittler, L.; Bischoff, S. (Hrsg.): Generali Engagementatlas 2015. Köln, 5-7.

Kummel, O.; Nadler, R. (2018): Die Grenzen des Ehrenamts. In: Informationen zur Raumentwicklung 6, 52-61.

Küpper, P.; Kundolf, S.; Mettenberger, T.; Tuitjer, G. (2018): Rural regeneration strategies for declining regions: trade-off between novelty and practicability. In: European Planning Studies 26, 2, 229-255. doi: 10.1080/09654313.2017.1361583 
Küpper, P.; Steinführer, A. (2017): Daseinsvorsorge in ländlichen Räumen zwischen Ausdünnung und Erweiterung: ein Beitrag zur Peripherisierungsdebatte. In: Europa Regional 23 (2015), 4, 44-60.

Landtag Brandenburg (2018): Zwischenbericht der EnqueteKommission 6/1 „Zukunft der ländlichen Regionen vor dem Hintergrund des demografischen Wandels". Potsdam. = Drucksache 6/8244.

Mai, R.; Swiaczny, F. (2008): Demographische Entwicklung: Potenziale für Bürgerschaftliches Engagement. Wiesbaden. = Materialien zur Bevölkerungswissenschaft 126.

Mayring, P. (1994): Qualitative Inhaltsanalyse. In: Boehm, A.; Mengel, A; Muhr, T. (Hrsg.): Texte verstehen: Konzepte, Methoden, Werkzeuge. Konstanz, 159-175. = Schriften zur Informationswissenschaft 14.

Moeritz, G. (2016): Vattenfall-Rückzug wird zur Last für die Lausitz. https://www.saechsische.de/vattenfall-rueckzug-wird-zur-lastfuer-die-lausitz-3312940.html (18.03.2020).

Nadler, R. (2017): The Elephant in the Room. Über das Verhältnis von demographischem Wandel, Daseinsvorsorge und zivilgesellschaftlichem Engagement in Deutschland. In: Raumforschung und Raumordnung | Spatial Research and Planning 75, 6, 449-512. doi: 10.1007/s13147-017-0507-y

o.V. (2018): Initiative ergreifen: Bürgerschaftliches Engagement in der Stadterneuerung. In: Polis. Magazin für Urban Development 25, 1, 26-29.

Olk, T.; Gensicke, T. (2014): Bürgerschaftliches Engagement in Ostdeutschland: Stand und Perspektiven. Wiesbaden. doi: 10.1007/978-3-658-03788-8

Patton, M. Q. (2002): Qualitative research and evaluation methods. Thousand Oaks.

Priller, E. (2016): Zivilgesellschaftliches Engagement. Eine Aufgabe für jede Nonprofit-Organisation und die Gesellschaft. In: Zimmer, A.; Hallmann, T. (Hrsg.): Nonprofit-Organisationen vor neuen Herausforderungen. Wiesbaden, 161-173. doi: 10.1007/978-3-658-10587-7 10

Rehm, H. (2008): Wissen und Ökonomie. In: RegioPol - Zeitschrift für Regionalwirtschaft 1, 3-9

Reim, D.; Schmithals, J. (2008): Kooperationsnetzwerke und Bürgerschaftliches Engagement als Erfolgsfaktoren für ostdeutsche Kommunen. In: Barlösius, E.; Neu, C. (Hrsg.): Peripherisierung - eine neue Form sozialer Ungleichheit? Berlin, 75-82. = Materialien der Berlin-Brandenburgischen Akademie der Wissenschaften 21

Simonson, J.; Ziegelmann, J. P.; Vogel, C.; Tesch-Römer, C. (2016): Zusammenfassung. Zentrale Ergebnisse des Deutschen Freiwilligensurveys 2014. In: Simonson, J.; Vogel, C.; TeschRömer, C. (Hrsg.): Freiwilliges Engagement in Deutschland. Der Deutsche Freiwilligensurvey 2014. Wiesbaden, 21-27. doi: 10.1007/978-3-658-12644-5_1

Sittler, L.; Amrhein, U.; Krimmer, H. (2015): Aus Wildwuchs eine Landschaft machen. In: Zeckra, C.; Amrhein, U.; Sittler, L.; Bischoff, S. (Hrsg.): Generali Engagementatlas 2015. Köln, 48-50.

Steffen, S. L.; Fothergill, A. (2009): 9/11 volunteerism: A pathway to personal healing and community engagement. In: The Social Science Journal 46, 1, 29-46. doi: 10.1016/j.soscij.2008.12.005
Steinführer, A.; Küpper, P.; Tautz, A. (2012): Gestaltung der Daseinsvorsorge in alternden und schrumpfenden Gemeinden - Anpassungs- und Bewältigungsstrategien im Harz. Braunschweig. $=$ Landbauforschung Sonderheft 367 .

Steinführer, A.; Moser, A. (2016): Vom Modernisierungsvehikel zur Überflüssigkeit? Soziale Infrastrukturen in ländlichen Räumen zwischen Leerfallen und Neuaneignung. In: Geographie und Landeskunde. Berichte 90, 4, 265-285.

Stölzel, F. (2019): Konnektivitäten von zivilgesellschaftlichen Netzwerkstrukturen: Eine Erhebung in Weißwasser und Forst. Masterarbeit (unveröffentlicht) an der Hochschule Zittau/ Görlitz.

Vogel, C.; Hagen, C.; Simonson, J.; Tesch-Römer, C. (2016): Freiwilliges Engagement und öffentliche gemeinschaftliche Aktivität. In: Simonson, J.; Vogel, C.; Tesch-Römer, C. (Hrsg.): Freiwilliges Engagement in Deutschland. Der Deutsche Freiwilligensurvey 2014. Wiesbaden, 91-150. doi: 10.1007/9783-658-12644-5_4

Willisch, A. (2011): Umbruch und Überleben. In: Bude, H.; Medicus, T.; Willisch, A. (Hrsg.): ÜberLeben im Umbruch: Am Beispiel Wittenberge: Ansichten einer fragmentierten Gesellschaft. Hamburg, 82-89.

Zimmer, A. (2010): Zivilgesellschaftsorganisationen - eine vernachlässigte Kategorie der Engagementforschung. In: Priller, E.; Alscher, M.; Dathe, D.; Speth, R. (Hrsg.): Zivilengagement: Herausforderungen für Gesellschaft, Politik und Wissenschaft. Berlin, 179-196. 